\title{
Análisis de la corrupción de funcionarios en la conducta ética de los servidores de la Administración Pública en el ejercicio de sus funciones: Región Ayacucho $2010^{1}$
}

Analysis of the Corruption of Officials in the Ethical Conduct of Public Administration

Servers in the Exercise of Their Functions: Ayacucho Region 2010

Patricia del Rosario Garay Peña ${ }^{2}$

http://dx.doi.org/10.21503/lex.v10i9.387

\section{Resumen}

Esta investigación tuvo como objetivo principal demostrar que el incremento de los actos de corrupción de funcionarios contribuye a la ineficiencia y la ineficacia de la Administración Pública. Es un estudio de tipo aplicativo, y el nivel es descriptivo y correlacional, puesto que se relacionan las medidas correctivas frente a la corrupción de funcionarios para que no se quiebre la confianza en el funcionamiento eficaz y eficiente de las instituciones públicas, así como el uso de un diseño metodológico de tipo no experimental y de corte transversal, porque tanto la corrupción de funcionarios como la ineficiencia de la Administración Pública se evidencian en la realidad, es decir, son observables, y por lo tanto no son de difícil demostración.

Palabras clave: corrupción de funcionarios y servidores públicos, servicios públicos, ineficiencia, ineficacia.

\begin{abstract}
This research had as main objective to demonstrate the increase in corruption by officials contributes to inefficiency and ineffectiveness of public administration, work that was done through the application type of research design, as will be apparent corrective measures in Officials of Corruption so as not to break the confidence in the effective and efficient functioning of public institutions and the use of a methodological design of non-experimental, cross sectional, because both the corruption of officials as inefficient Public Administration in the reality show, are observable, and therefore are not difficult to show.
\end{abstract}

Key words: corruption of officials and public servants, public services, inefficiency, ineffectiveness.

\footnotetext{
Esta tesis puede ser revisada en la biblioteca de la Universidad Alas Peruanas.

2 Título de abogada por la Universidad Alas Peruanas.
} 


\section{Introducción}

Siendo la corrupción un problema que afecta a todos los niveles de la sociedad y a los diferentes niveles de gobierno -nacional, regional y local-, se parte del análisis de los factores que desencadenan esta problemática, teniendo como herramientas la doctrina, la legislación nacional e internacional, los diferentes casos investigados y la ayuda de diversas personas ligadas al tema, mediante las cuales se ha podido alcanzar el propósito trazado.

Por tanto, es el objetivo principal de esta investigación dar a conocer las causas y los efectos de la corrupción de funcionarios que contribuyen a la ineficiencia e ineficacia de la Administración Pública, con el propósito de proporcionar un mejor enfoque del problema que afecta desde hace un buen tiempo a la población del distrito de Ayacucho, donde el 60\% de ciudadanos se reconocen corruptos, el 81\% desaprueba la gestión de su Alcalde Provincial y el 79\% desaprueba la gestión pública de su Presidente Regional.

La investigación es importante porque nos permitirá analizar este hecho social y su evolución dentro de la sociedad, identificando los distintos problemas que afectan su desarrollo armonioso dentro del ámbito del distrito de Ayacucho y saber qué tipos de acciones existen por parte del Estado y del propio Derecho para hacerle frente.

En la investigación, se busca determinar los aspectos a través de los cuales se ha podido entender las causas y los efectos de los actos ilícitos que cometieron los funcionarios y servidores públicos y los daños que pueden acarrear tanto en la sociedad como en la propia institución pública en la cual laboran. Se parte de la recopilación de datos que lo sustentan, buscando que contrastar opiniones y diferentes enfoques de autores que han escrito sobre el problema, generando una posición, en la investigadora, y desde este análisis proponer mecanismos de solución. Se han obtenido resultados a través de las técnicas e instrumentos de recolección de datos. La aplicación de los instrumentos y técnicas usados en la búsqueda de los resultados ha dado una visión más amplia del creciente y problemático fenómeno llamado corrupción de funcionarios y servidores en la Administración Pública. Finalmente, se han presentado las conclusiones, recomendaciones.

\section{Objetivo general}

- Demostrar que el incremento de los actos de corrupción de funcionarios contribuye a la ineficiencia de la Administración Pública a través del análisis de los diferentes procesos existentes al interior de las Procuradurías Públicas Anticorrupción Descentralizadas, con el fin de promover la creación de políticas públicas articuladas y fortalecidas que permitan devolver la confianza en una administración objetiva, imparcial, eficaz y transparente. 


\section{Objetivos específicos}

- Identificar cómo el servidor público en ejercicio de sus funciones es influenciado por los factores sociales y políticos que estimulan los actos de corrupción dentro de la función pública, con el fin de confirmar su percepción de la misma como un medio de enriquecimiento personal, prescindiendo de las normas y reglas establecidas.

- Señalar la relación que existe entre el beneficio pecuniario como medio para agilizar los trámites administrativos y el patrimonio del Estado, que se ve afectado en el incremento de sus fondos públicos con el propósito de indicar que existe una correlación negativa entre crecimiento y altos niveles de corrupción.

- Determinar que las causas de una actuación ilegítima del funcionario público son sancionadas en principio a través de la Ley de Procedimiento Administrativo General, con el fin de establecer la responsabilidad de la Administración Pública y del personal a su servicio.

\section{Hipótesis y variables}

Si los actos de corrupción de funcionarios continúan incrementándose como un medio para agilizar los trámites en las instituciones del Estado, entonces se desnaturalizará el objetivo de la Administración Pública hacia los administrados.

\section{Hipótesis específicas}

- Si el servidor público en ejercicio de sus funciones continúa incumpliendo intencionalmente el principio de imparcialidad y abuso de poder con tal de obtener un beneficio propio, entonces la función pública perderá su esencia natural de atender los intereses públicos de la Nación.

- Si el incentivo de un beneficio pecuniario se convierte en práctica corriente para que el funcionario realice actos que excedan la regla, entonces perjudicará el ingreso de activos al patrimonio del Estado, generando con ello que menos inversionistas quieran invertir en el país.

- Toda actuación ilegitima del funcionario público será sancionada bajo la Ley de Procedimiento Administrativo General, en cuanto a su responsabilidad dentro de la Administración Pública.

Conceptos importantes

- Administración Pública. Conjunto de ideas, actitudes, normas, procesos, instituciones y 
otras formas de conducta humana que determinan cómo se distribuye y ejerce la autoridad política y cómo se atiende los intereses públicos.

- Clientelismo Político. Es un sistema extraoficial de intercambio de favores, en el cual los titulares de cargos políticos regulan la concesión de prestaciones, obtenidas a través de su función pública o de contactos relacionados con ella, a cambio de apoyo electoral.

- Corrupción de funcionarios. Es toda aquella acción u omisión del servidor público que lo lleva a desviarse de los deberes formales de su cargo con el objeto de obtener beneficios pecuniarios, políticos o de posición social, así como cualquier utilización en beneficio personal o político de información privilegiada, influencias u oportunidades.

- Eficacia. Capacidad para obrar o para conseguir un resultado determinado; está referida a los resultados con relación a las metas y cumplimiento de los objetivos organizacionales, por eso para ser eficaz se debe priorizar las tareas y realizar en orden de procedencia aquellas que contribuyen a alcanzar los objetivos y metas previstas, por lo que se debe asegurar que lo que se haga valga la pena y conduzca a un fin.

- Eficiencia. Capacidad para lograr un fin empleando los mejores medios posibles; es decir, realizar un trabajo o una actividad al menor costo posible y en el menor tiempo, sin desperdiciar recursos económicos, materiales y humanos, pero a la vez implica calidad al realizar bien lo que se espera lograr.

- Empleado público. Todo funcionario o servidor de las entidades de la Administración Pública, en cualquiera de los niveles jerárquicos sea éste nombrado, contratado, designado, de confianza o electo que desempeñe actividades o funciones en nombre o al servicio del Estado, no importando el régimen jurídico de la entidad en la que preste servicios ni el régimen laboral o de contratación al que esté sujeto.

- Función Pública. Concebida como la práctica concreta en la cual deben intervenir el Gobierno, los trabajadores públicos (servidores y funcionarios) y el resto de la Colectividad Nacional, en base a los principios de eficacia social, compromiso y participación.

- Funcionario público. Sujeto elegido o designado principalmente para desempeñar cargos políticos o de confianza, sin concurso. Representa al Estado y tiene poder de decisión, expide resoluciones, otorga instrumentos públicos, etc.

- Servidor público. Sujeto conocido como "empleado", "empleado público", "trabajador público", "trabajador estatal", etc., que ingresa a la Administración Pública bajo ciertos requisitos y que está sometido a la Carrera Administrativa o que tienen la condición de eventual. Este sujeto no tiene la facultades propias de un funcionario público en el 
ejercicio de sus funciones, generalmente está bajo las órdenes directas o indirectas de un funcionario.

\section{Metodología}

\section{Tipo de investigación}

De tipo aplicativo, porque se darán medidas correctivas frente a la corrupción de funcionarios, utilizando los principios doctrinarios jurídicos para que no se quiebre la confianza en el funcionamiento eficaz y eficiente de las instituciones públicas.

\section{Diseño metodológico}

Es no experimental porque tanto la corrupción de funcionarios como la ineficiencia de la Administración Pública se evidencian en la realidad, es decir, son observables, y por lo tanto no son de difícil demostración. Así mismo es de corte transversal, puesto que para realizar un mejor estudio fue necesario precisar el campo y el año en donde se realizaría la investigación y por lo tanto se tomó como referencia el año 2010 y el Gobierno Regional de Ayacucho.

Niveles de investigación

Descriptivo. Porque la problemática generada por la corrupción de funcionarios ha determinado que paulatinamente este fenómeno se vaya institucionalizando, poniendo en riesgo el control y manejo adecuado de las instituciones públicas.

Correlacional. Porque existe una relación directa entre la corrupción de funcionarios y la Administración Pública, dado que mientras más aumenta la corrupción de funcionarios habrá mayor desconfianza en la conducta transparente y eficaz que debe desarrollar el funcionario público en el ejercicio de sus funciones.

\section{Métodos}

Inductivo. Porque a partir de la corrupción de funcionarios se pueden presentar los siguientes problemas:

- Desconfianza en la Administración Pública.

- Pérdida de control y manejo de las instituciones públicas.

- Abuso por parte de los funcionarios públicos.

- Debilitamiento de la legitimidad del gobierno.

- Ineficiencia en el aprovechamiento de los recursos.

- Consolidación de la desigualdad social. 
Deductivo. Frente a los problemas planteados de la corrupción de funcionarios, se concluye que: es imprescindible un mínimo consenso político frente a la lucha eficaz contra la corrupción, dotando de legitimidad a las políticas públicas que se implementen, otorgándoles continuidad a las mismas por encima de las coyunturas particulares y garantizando total independencia a los órganos encargados de la fiscalización y la justicia.

Estadistico. Al comparar las cifras de estadística a nivel mundial en cuanto a la corrupción de funcionarios, se observa que en el reporte global de los 69 países y territorios que participaron en la encuesta que Transparencia Internacional realizó entre octubre del 2008 y febrero del 2009 muestra algunas particularidades de Perú respecto a América Latina y el mundo.

Analítico. Frente al fenómeno de la corrupción se observaron las diferentes causas como la deficiencia en los sistemas de control y el desinterés del administrado para denunciar, así como la distribución política del poder en la Administración Pública concentrada y sin transparencia que desde la época de la República se han venido acentuando en el país.

\section{Población y muestra}

\section{Población constituida por la siguiente Unidad de Análisis:}

- Expedientes que contienen casos de corrupción a nivel de todo el distrito judicial de Ayacucho, que comprende parte del valle de los ríos Apurímac y Ene-VRAE

- Expedientes que contienen casos en el poder judicial de Ayacucho por peculado y malversación de fondos.

- 83 funcionarios y servidores procesados por delitos contra la Administración Pública.

- 317 empleados públicos del Gobierno Regional de Ayacucho

El total de población se encuentra constituida por un total de 400 empleados públicos.

Muestra: constituida por la siguiente Unidad de Análisis.

Para la obtención de la muestra, se utilizó el procedimiento de muestreo probabilístico aleatorio simple, puesto que todas las unidades de observación tienen las mismas probabilidades de formar parte de la muestra, la cual fue aplicada a los 400 empleados públicos.

Para determinar el tamaño de la misma se recurre a la ecuación para cálculo muestral, como se indica:

$$
\mathrm{n}=\frac{\mathrm{Z}^{2} \times(\mathrm{p} \times \mathrm{q} \times \mathrm{N})}{\mathrm{E}^{2} \times(\mathrm{N}-1)+\mathrm{Z}^{2} \times \mathrm{p} \times \mathrm{q}}
$$


Donde:

Z: Desviación Estándar según el nivel de confianza $(Z=1,96)$

E: Margen de error $(10 \%=0,1)$

p: Probabilidad de ocurrencia de los casos $(\mathrm{p}=0,8)$

q: Probabilidad de no ocurrencia de los casos ( $\mathrm{q}=0,2)$

$\mathrm{N}$ : Tamaño del Universo $(\mathrm{N}=400)$

n: Tamaño óptimo de la muestra

$$
n=\frac{(1,96)^{2} \times(0,8 \times 0,2 \times 400)}{(0,1)^{2} \times(400-1)+(1,96)^{2} \times 0,8 \times 0,2}
$$

\section{$\mathrm{n}=53$}

Por tanto, la muestra queda constituida por 53 empleados públicos que laboran en la ciudad de Huamanga, Ayacucho.

Técnicas e instrumentos de recolección de datos

Tipos de técnicas e instrumentos. Dado que el estudio supone el inicio de investigaciones futuras, se utilizarán en esta investigación las siguientes técnicas:

- Encuesta

- Entrevista

- Análisis documental

Este conjunto de técnicas permitió recoger información considerada pertinente para abordar los objetivos del presente trabajo.

Instrumentos. Los principales instrumentos diseñados para la presente investigación son:

- Cuestionario

- Guía de Entrevista

Guía de Análisis Documental 


\section{Tablas y análisis de datos}

Actualmente a través de las estadísticas sobre la corrupción según Transparencia Internacional (TI), que es una organización no gubernamental, se puede conocer la posición de los países en función a los indicadores de corrupción. El Barómetro Global de la Corrupción 2009 de TI presenta las principales conclusiones de una encuesta de opinión que analiza las opiniones del público en general sobre corrupción, así como sus experiencias de soborno alrededor del mundo. Evalúa en qué grado se percibe a las instituciones y los servicios públicos claves como corruptos, mide la opinión de los ciudadanos sobre las iniciativas del gobierno contra la corrupción e incluye preguntas indagatorias sobre el nivel de captura del Estado y la predisposición de las personas a pagar una cantidad adicional a las empresas con conducta transparente.

Con este propósito, el Barómetro Global de la Corrupción 2009 entrevistó a 73,132 personas en 69 países entre octubre de 2008 y febrero de 2009, cuya población se presenta por región y en orden alfabético en la Tabla 1. 
Tabla 1.Clasificación regional

\begin{tabular}{|c|c|c|}
\hline \multicolumn{3}{|c|}{ CLASIFICACIÓN REGIONAL } \\
\hline UE+ Islandia, Israel, Noruega y Suiza & Asia Pacífico & África Subsahariana \\
\hline $\begin{array}{ll}\text { - } & \text { Austria } \\
\text { - } & \text { Bulgaria } \\
\text { - } & \text { Dinamarica Checa } \\
\text { - } & \text { Finlandia } \\
\text { - } & \text { Grecia } \\
\text { - } & \text { Islangría } \\
\text { - } & \text { Israel } \\
\text { - } & \text { Italia } \\
\text { - } & \text { Lituania } \\
\text { - } & \text { Países Bajos } \\
\text { - } & \text { Poruega } \\
\text { - } & \text { Portugal } \\
\text { - } & \text { Rumania } \\
\text { - } & \text { Suizpaña } \\
\text { - } & \text { Reino Unido }\end{array}$ & $\begin{array}{ll}\text { - } & \text { Brunei Darussalam } \\
\text { - } & \text { Honboya Kong } \\
\text { - } & \text { India } \\
\text { - } & \text { Indonesia } \\
\text { - } & \text { Japón } \\
\text { - } & \text { Corea del Sur } \\
\text { - } & \text { Malasia } \\
\text { - } & \text { Pakistán } \\
\text { - } & \text { Filipinas } \\
\text { - } & \text { Singapur } \\
\text { - Tailandia }\end{array}$ & $\begin{array}{ll}\text { - } & \text { Camerún } \\
\text { - } & \text { Ghana } \\
\text { - } & \text { Kenia } \\
\text { - } & \text { Liberia } \\
\text { - } & \text { Nigeria } \\
\text { - } & \text { Senegal } \\
\text { - } & \text { Sierra Leona } \\
\text { - } & \text { Uganda } \\
\text { - } & \text { Zambia }\end{array}$ \\
\hline Balcanes occidentales + Turquía & $\begin{array}{c}\text { Nuevos Estados Independientes } \\
\text { (NEI)+ Mongolia }\end{array}$ & $\begin{array}{c}\text { Medio Oriente y África del } \\
\text { Norte }\end{array}$ \\
\hline $\begin{array}{ll}\text { - } & \text { Bosnia y Herzegovina } \\
\text { - } & \text { Croacia } \\
\text { - } & \text { Kosovo } \\
\text { - } & \text { Serbia } \\
\text { - } & \text { Turquía }\end{array}$ & $\begin{array}{ll}\text { - } & \text { Armenia } \\
\text { - } & \text { Azerbaiyán } \\
\text { - } & \text { Bielorrusia } \\
\text { - } & \text { Georgia } \\
\text { - } & \text { Moldavia } \\
\text { - } & \text { Rongolia } \\
\text { - } & \text { Ucrania }\end{array}$ & $\begin{array}{ll}\text { - } & \text { Irak } \\
\text { - } & \text { Kuwait } \\
\text { - } & \text { Líbano } \\
\text { - } & \text { Marruecos }\end{array}$ \\
\hline \multicolumn{2}{|l|}{ América del Norte } & América Latina \\
\hline $\begin{array}{l}\text { - } \quad \text { Canadá } \\
\text { - } \quad \text { Estados Unidos }\end{array}$ & $\begin{array}{ll}\text { - } & \text { Argentina } \\
\text { - } & \text { Bolivia } \\
\text { - } & \text { Chile } \\
\text { - } & \text { Colombia } \\
\text { - } & \text { El Salvador } \\
\text { - } & \text { Panamá } \\
\text { - } & \text { Perú } \\
\text { - } & \text { Venezuela }\end{array}$ & \\
\hline
\end{tabular}

Fuente: Barómetro Global de la Corrupción 2009 de Transparency International

A nivel mundial, los encuestados percibían a los partidos políticos como la institución nacional más corrupta, seguida de cerca por la administración pública. 
A nivel de América Latina, el Índice de Percepción de la Corrupción (IPC) elaborado por Transparencia Internacional y que varía entre 0 y 10, muestra que la mayor percepción de la corrupción se presenta en un país, cuanto más cercano a 10 se encuentre el índice. $\mathrm{Al}$ respecto se aprecia que los valores de este índice en el 2001, resultaron en 7,5 en Chile, 5.1 en Uruguay y 4,1 en Perú, países que tendrían la mayor percepción de la corrupción. En el lado opuesto Bolivia, Ecuador y Venezuela son los países que de acuerdo con este índice se presentan como aquellos países donde la percepción de corrupción fue más baja.

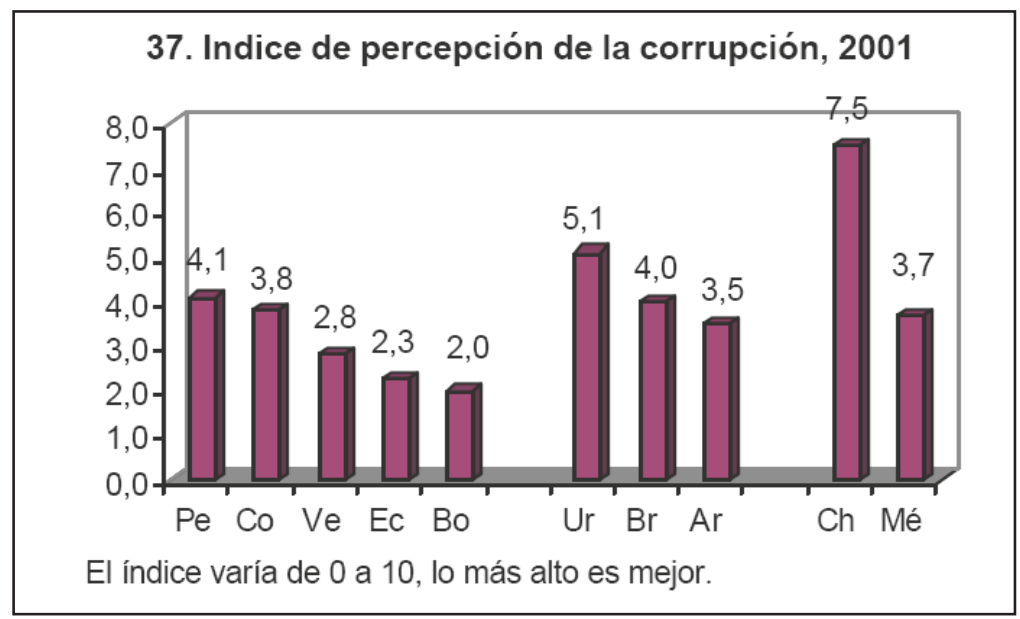

Fuente: Comunidad Andina.

Figura 1. Índice de percepción de la corrupción 2001

En cuanto al Perú, se realizó la Quinta Encuesta Nacional sobre Corrupción 2008 y Proética solicitó a Ipsos Apoyo Opinión y Mercado la realización de un estudio cuantitativo para determinar la percepción de la ciudadanía sobre la corrupción y comparar los resultados con los obtenidos en la medición de los años 2006 (segundo gobierno de Alan García) y 2004 (gobierno de Alejandro Toledo Manrique). Para tal efecto, se realizó una encuesta entre jefes de hogar de todos los niveles socioeconómicos y en todos los departamentos del país.

Uno de los principales hallazgos que reveló la investigación fue la que se halló cuando se preguntó a los entrevistados (5790 personas) sobre su percepción de la corrupción en el país. (http://www.proetica.org.pe/convenios.php?llave $=5 \& M m e n u=2,3$, Fecha de consulta: agosto 28 de 2010) 


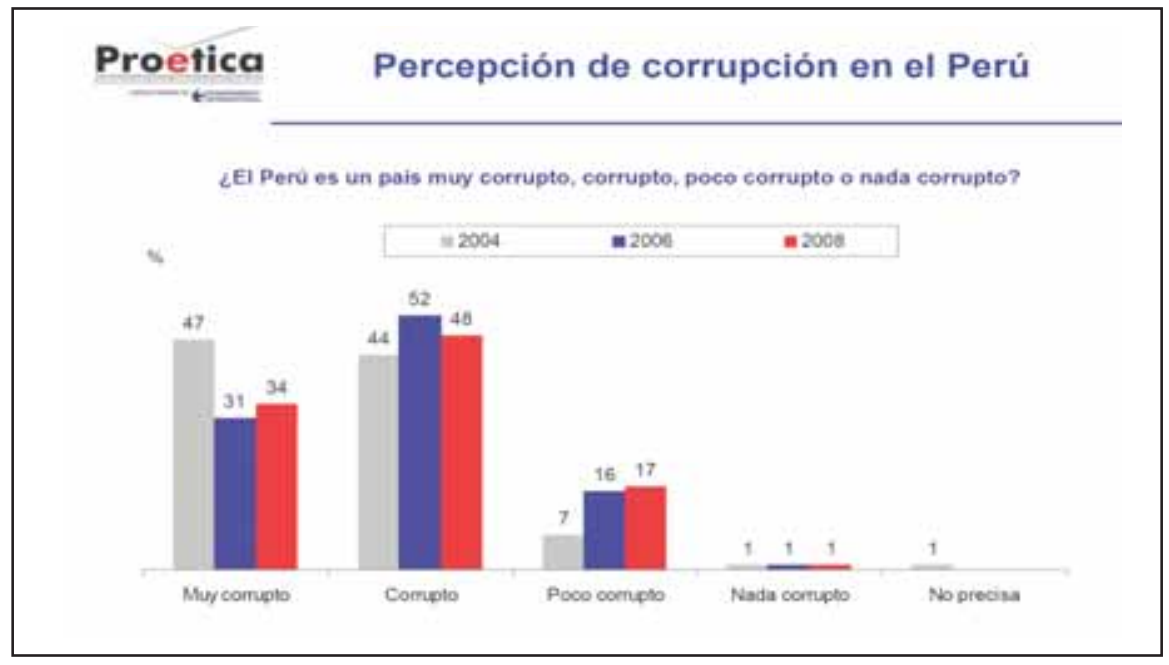

Fuente: Proética. Consejo Nacional para la Ética Pública.

Figura 2. Percepción de corrupción en el Perú

- En el 2004 la percepción del país como "muy corrupto” presentó su pico más alto con un 47\% durante el gobierno de Alejandro Toledo Manrique, respecto a un 31\% en el 2006, lo cual reflejó un índice a la baja de 16 puntos porcentuales, retomando una subida de 3 puntos porcentuales en el 2008.

- La percepción del país como “corrupto” mostró su pico más alto en el 2006 con un 52\%, año en el que finalizaba el gobierno de Alejandro Toledo y asumía el poder el 28 de julio de 2006 Alan García Pérez, bajando a un 48\% dicha percepción.

- Del total de entrevistados, un 17\% lo percibió como “poco corrupto" en el 2008, 16\% para el 2006 y 7\% en el 2004; con lo cual se percibe un considerable aumento entre el 2004 y el 2008.

Puede apreciarse, por tanto, que la población peruana considera al Perú como un país corrupto, donde la ola de acontecimientos producidos en los últimos años señala la ocurrencia permanente de prácticas delictivas que comprometen a la administración pública y que arrastra las consecuencias de la inacción, la ineficiencia y la corrupción de funcionarios que usan mal los fondos públicos siendo afectado de esta manera el desarrollo del país y el bienestar de la población. A ello debe sumarse la cuestionada actuación de ciertos personajes del ámbito político, envueltos en denuncias escandalosas, cuyas decisiones dejan una percepción negativa en la opinión pública. 


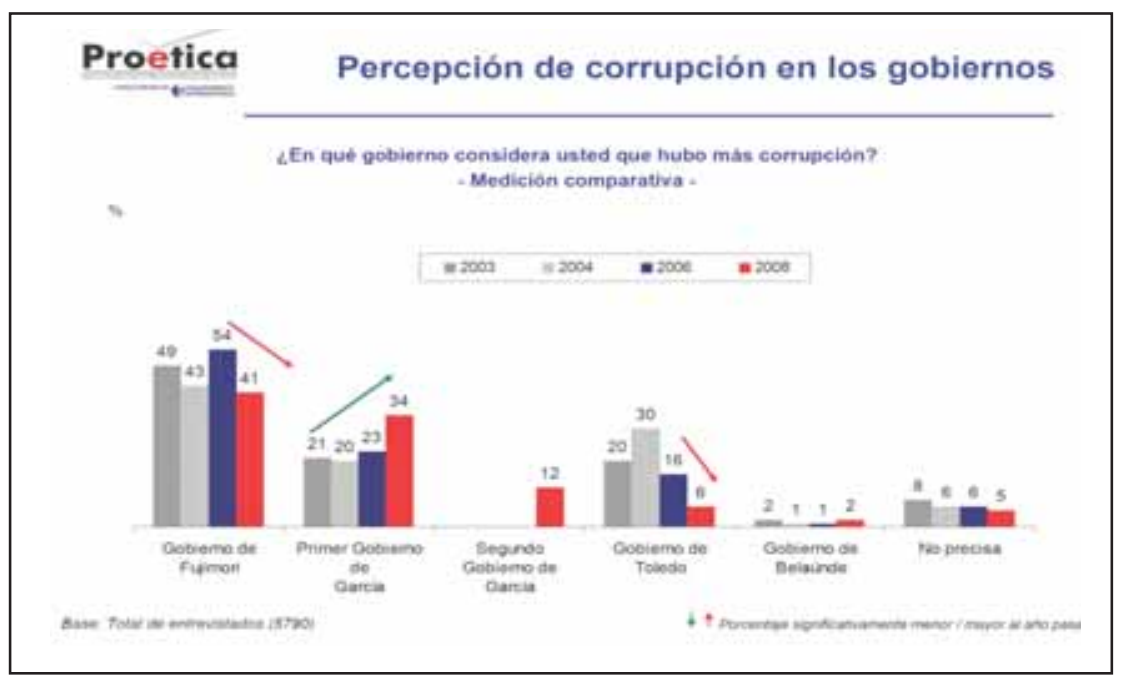

Fuente: Proética. Consejo Nacional para la Ética Pública.

\section{Figura 3. Percepción de corrupción en los gobiernos}

En cuanto a la percepción del nivel de corrupción de los últimos seis gobiernos que tuvo el país, muestran al gobierno de Fujimori como el más corrupto, pese a notarse una disminución de más de diez puntos con respecto a la medición anterior.

Sin embargo, a pesar del problema existente, se puede controlar dicho problema, si se toman las siguientes medidas:

- Es imprescindible un mínimo consenso político favorable a esta causa. Este contexto no solo dota de legitimidad a las políticas que se implementen sino que otorga continuidad a las mismas por encima de las coyunturas particulares.

- Garantizar una suficiente independencia interna (dentro de su propio aparato institucional) y externa (respecto de influencias del poder político o económico de turno) de los órganos responsables del sistema de administración de justicia (Ministerio Público, Poder Judicial y Policía Nacional del Perú).

- Evitar la concentración de funciones y de poderes discrecionales en los funcionarios públicos. 


\section{Conclusiones}

1) Queda demostrada categóricamente la hipótesis general a través de los datos obtenidos, identificándose que si los actos de corrupción de funcionarios continúan incrementándose como un medio para agilizar los trámites en las instituciones del Estado, entonces se desnaturalizará el objetivo de la administración pública hacia los administrados.

2) El $75,47 \%$ de los encuestados indica que los trámites excesivos en la administración pública se perciben como un factor que contribuye a que el administrado se vea en la necesidad de corromper al funcionario o servidor público, puesto que cuando se habla en términos de celeridad y eficiencia, éstos sólo funcionan cuando el empleado público recibe un beneficio, generalmente pecuniario, para realizar su labor transparente y honesta que por ley está obligado a realizar.

3) Todas las estrategias destinadas al control de la corrupción dentro de la administración pública no consiguen hasta el momento promover el mejoramiento de la gestión pública, puesto que el Estado, las instituciones a cargo y la misma población no trabajan de una manera articulada y con total transparencia; de tal manera que los ciudadanos expresan un requerimiento constante de aplicar las técnicas de gestión modernas para que contribuyan significativamente a la resolución de problemas y conflictos que la gestión pública enfrenta en sus distintos niveles.

4) El 52,83\% de la población encuestada percibe que el funcionario o servidor público carece de ética y acepta un soborno por la falta de valores y una adecuada educación que permita contar dentro de la administración pública con empleados públicos idóneos y probos que tengan un pleno conocimiento del aspecto deontológico y básicamente del Código de Ética y la debida aplicación de los principios, deberes y prohibiciones establecidos en el mismo.

5) El marco regulatorio que se tiene en la administración pública para el tema de las sanciones no se aprecia como el adecuado, situación que se percibe como una manifestación concreta de la indiferencia real del poder político con los verdaderos propósitos de erradicar los actos de corrupción dentro de las entidades estatales al no fortalecer en forma debida y urgente a los órganos estatales de las labores de control y coerción como son las Procuradurías Públicas, la Contraloría General de la República, el Ministerio Público, el Poder Judicial, etc.

6) El $77,36 \%$ de la población encuestada no tiene conocimiento de lo que es un manual sectorial, hecho que suscita gran preocupación, puesto que el manual es el instrumento que proporciona los procesos de formulación, redefinición y armonización de políticas 
y agendas sectoriales en las instituciones públicas, dirigido al fortalecimiento de la capacidad técnico-operativa de las instituciones para enfrentar el reto de la planificación en consonancia con las directrices emanadas del Plan Nacional de Desarrollo y del nuevo marco constitucional del Estado.

\section{Recomendaciones}

1) A las autoridades del sector público encargadas de seleccionar, evaluar y contratar a quienes opten por la carrera administrativa se les recomienda que ante los diferentes casos de corrupción garantizar con absoluta transparencia todos esos procesos que tienen por objetivo permitir la incorporación de personal idóneo, garantizando su permanencia y asegurar su desarrollo y promover su realización personal en el desempeño del servicio público ubicándolos según su calificación y méritos.

2) Al Poder Judicial se recomienda que se hace necesario garantizar el respeto a las garantías procesales durante el proceso al imputado de la comisión de los delitos contra la Administración Pública, siendo totalmente transparente e imparcial al momento de aplicar la sanción correspondiente bajo los principios de razonabilidad y proporcionalidad.

3) Al Consejo de Defensa Jurídica del Estado se le recomienda que dote de la infraestructura y el personal necesario a las Procuradurías Públicas Anticorrupción Descentralizadas para que la labor tan importante que realizan no se vean disminuidas por la carencia de recursos humanos y logística que no permitan un adecuado desenvolvimiento de su función en representación del Estado.

4) $\mathrm{Al}$ Poder Ejecutivo, promover en todos los niveles y estructuras de cada entidad pública el Código de Ética de la Función Pública, Ley $N^{\circ}$ 27815, bajo el principio que los funcionarios y empleados públicos son servidores exclusivos de los intereses de la colectividad y no de parcialidad, de partido político o interés particular alguno. Así como implementar dentro de toda institución pública un órgano que establezca un código ético de base y se encargue de su promoción constante y que a su vez logre como objetivo principal la formación ética de los empleados públicos que ha de ser permanente.

5) A los entes rectores de las universidades peruanas, se recomienda la implantación en el programa curricular de todas sus facultades, en especial las facultades de Derecho, el curso de Deontología o Ética Jurídica que permita sentar y reafirmar los valores que cada alumno trae consigo desde su hogar. 
6) A la población en su conjunto, se le recomienda actuar de manera decidida ante los actos ilícitos que cometan los funcionarios o servidores públicos, dejando de lado los temores a denunciar, la indiferencia y prejuicios como que los responsables nunca son perseguidos. Si no existe un compromiso real y efectivo de la ciudadanía con las autoridades a nivel de cada uno de los gobiernos seguirá institucionalizándose la corrupción y se perderá el control y el manejo de las instituciones públicas.

\section{Referencias}

- Asociación Peruana de Derecho Administrativo (2010). Modernizando el Estado para un pais mejor. Lima: Palestra Editores, 855 pp.

- Cabanellas de Torres, G. (2006). Diccionario Jurídico Elemental. Buenos Aires, Argentina: Editorial Heliasta, 422 pp.

- Camou, A., (2001).Los desafíos de la gobernabilidad (Estudio preliminar y compilación). Primera Edición, México: Flacso/IISUNAM/, 341pp.

- Chanamé Orbe, R. (2006). Diccionario Jurídico Moderno. 4ª Edición. Lima, Perú: Abogado Editores E.I.R.L., 497 pp.

- Enríquez Rubio, E. (2002).Administración de recursos materiales en el sector público. México: Instituto Nacional de Administración Pública A.C., 605 pp.

- Flores Polo, P. (2002).Diccionario Jurídico Fundamental. 2da. Edición. Lima, Perú: Editora Jurídica Grijley, 422 pp.

- Frisancho Aparicio, M. (2002).Delitos contra la Administración Pública. 2da. Edición. Lima, Perú: Editora Fecal, 762 pp.

- Galeano, E. (1984).Las venas abiertas de América Latina. 38 Edición. México: Siglo Veintiuno Editores S.A.,470 pp.

- Morón Urbina, J. (2007).Comentarios a la Ley del Procedimiento Administrativo General. 6a Edición, Lima, Perú: Gaceta Jurídica S.A., 854 pp.

- Mory Príncipe, F. (2009).El Proceso Administrativo Disciplinario. 4ta. Edición. Lima, Perú: Editorial Rhodas, 714 pp.

- Patrón Faura, P. (2004). Derecho Administrativo y Administración Pública en el Perú. 8va. Edición. Lima, Perú: Editora Jurídica Grijley, 637pp. 
- Prats, J. (2001). Gobernabilidad democrática para el desarrollo humano. Marco conceptual y analítico. Instituciones y Desarrollo, No 10, IIG/PNUD/Generalitat de Catalunya, 148 pp.

- Retamozo Linares, A. (2000). Responsabilidad civil del Estado por corrupción de funcionarios públicos. Lima, Perú: Editores N\&S, 241 pp.

- Rubio Correa, M. (2006). El sistema jurídico. Sexta reimpresión. Lima, Perú: Fondo Editorial de la Pontificia Universal Católica del Perú, 384 pp. 